\title{
Automated Vocabulary Instruction in a Reading Tutor
}

\author{
Cecily Heiner ${ }^{1,2}$, Joseph Beck ${ }^{2}$, and Jack Mostow ${ }^{2}$ \\ ${ }^{1}$ School of Computing, University of Utah, Salt Lake City, UT USA 84112 \\ cecily@cs.utah.edu \\ Tel.: 801-792-4352 \\ ${ }^{2}$ School of Computer Science, Carnegie Mellon University, Pittsburgh, PA 15213 \\ joseph.beck@cmu.edu, mostow@cs.cmu.edu
}

\begin{abstract}
This paper presents a within-subject, randomized experiment to compare automated interventions for teaching vocabulary to young readers using Project LISTEN's Reading Tutor. The experiment compared three conditions: no explicit instruction, a quick definition, and a quick definition plus a poststory battery of extended instruction based on a published instructional sequence for human teachers. A month long study with elementary school children indicates that the quick instruction, which lasts about seven seconds, has immediate effects on learning gains that did not persist. Extended instruction which lasted about thirty seconds longer than the quick instruction had a persistent effect and produced gains on a posttest one week later.
\end{abstract}

\section{Introduction}

Many tutorial domains require learning vocabulary. We present an experimental comparison of three ways to introduce new words. In this paper, we automate a published sequence for human-administered vocabulary instruction and evaluate the effectiveness of the automated sequence compared. As baselines, we use a null treatment with no instruction, and a quick treatment consisting of exposure to the definition. For the quick treatment, we included the definition of the word as part of the story text, immediately after the sentence containing the vocabulary word. For third condition, we included the same instruction as the quick treatment plus extended instruction at the end of the story. The extended instruction comes from experts in vocabulary instruction [1] who suggest the following steps for classroom teaching:

1) Give the context of the vocabulary word in the story. The Reading Tutor displayed and read a sentence of the form "In this story, ..." followed by the story sentence containing the vocabulary word.

2) Repeat the word to create a phonological representation of the word. The Reading Tutor displayed the word, read the word, and asked the student to repeat the word.

3) Explain the meaning of the word. The Reading Tutor displayed and read a sentence with the vocabulary word followed by "means" followed by the definition.

4) Provide examples of the word in other contexts outside the story. The Reading Tutor displayed and read one additional hand-crafted sentence containing the vocabulary word. 
5) Allow the children to practice the word in new contexts. The Reading Tutor displayed and read another hand-crafted sentence containing the vocabulary word and asked "Does this make sense?". The student could click on the words "yes" and "no" to respond, but received no feedback.

6) Say the word again to reinforce the phonological representation. The reading Tutor displayed the word, read the word, and asked the student to repeat the word.

We will call these six steps of vocabulary instruction the Beck battery.

Each story contained three selected vocabulary words with each of the three words assigned randomly to a different treatment condition: null, quick, and Beck battery. In the Null condition, the student simply encountered the word in context without instruction. In the Quick condition, students received in-story instruction in the form of a handcrafted definition after the sentence where the vocabulary word first appears. On the word assigned to the Beck condition, students received the same kind of handcrafted definition as in the quick condition plus the previously described extended instruction based on the Beck battery.

Each student was tested on each word three times: in a pretest, in a posttest, and in a delayed posttest. The pretest contained three questions that required matching a vocabulary word to its definition. In addition to the same matching questions on the pretest, the immediate posttest and delayed posttest contained cloze (fill in the blank) questions. This project is implemented in the context of Project LISTEN's Reading Tutor which uses automatic speech recognition to assist children (mostly ages 6-10) in reading aloud.

\section{Evaluation}

Students used Project LISTEN's Reading Tutor [2] during July 2005 reading clinics at two elementary schools. We analyzed trials where the student had completed all of the items in the pretest, the immediate posttest, and the delayed posttest for all three words in a story. This left a dataset with fourteen students who had read a total of eighteen stories (four distinct texts) for a total of eighteen trials. In each of the conditions with instruction, the students answered three more matching questions correctly on the Immediate Posttest than they did on the pretest, but only Beck instruction resulted in gains that lasted a week or longer on the matching task (five questions). With the cloze questions, the gains were even more remarkable; students who received Beck instruction answered almost twice as many delayed posttest questions correctly compared to other conditions (ten instead of six).

We used a logistical regression in SPSS to test statistical significance because the data did not have a normal distribution and trials were correlated [3]. The dependent variable was whether or not a posttest item was correct. The other factors were the delay, the treatment, the question type, the performance on pretest, and the interaction between treatment and delay. One output of a logistical regression is a set p-values that represent the probability that the results happened by chance. The question type and delay were both statistically insignificant predictors. Beck instruction was more effective than no instruction $(\mathrm{p}=0.058)$. Beck instruction was also more effective than quick instruction. Whether the student received Beck instruction was a stronger predictor of posttest performance than whether the pretest question was correct. 


\section{Acknowledgements}

This work was partially supported by the National Science Foundation, under ITR/IERI Grant No. REC-0326153. Any opinions, findings, and conclusions or recommendations expressed in this publication are those of the author(s) and do not necessarily reflect the views of the National Science Foundation or the official policies, either expressed or implied, of the sponsors or of the United States Government.

\section{References}

1. Beck, I.L., M.G. McKeown, and L. Kucan, Bringing Words to Life. 2002: The Guilford Press.

2. Mostow, J. and G. Aist, Evaluating tutors that listen: An overview of Project LISTEN, in Smart Machines in Education, K. Forbus and P. Feltovich, Editors. 2001, MIT/AAAI Press: Menlo Park, CA. p. 169-234.

3. Menard, S., Applied Logistic Regression Analysis. Quantitative Applications in the Social Sciences, 1995. 106. 